\title{
Resposta da creatina quinase em diferentes volumes de treinamento resistido em circuito com indivíduos treinados e destreinados
}

\author{
Response of creatine kinase in different volumes of resistance training in circuit with trained and \\ untrained
}

Respuesta de la creatina quinasa en diferentes volúmenes de entrenamiento de resistencia en circuito con entrenados y desentrenados

\section{Resumo}

Objetivo: Analisar a cinética da creatina quinase $(\mathrm{CK})$ e suas concentrações no treinamento resistido em circuito, com diferentes volumes de um peso fixo de vinte repetições máximas (20RM), para homens treinados (TR) e destreinados (DT). Método: Compuseram a amostra, por voluntariado, 18 homens (9 voluntários TR e 9 DT), com idade entre 18 e 35 anos. Após receberem informações sobre o projeto, os voluntários, realizaram uma avaliação física, e posteriormente testes e retestes de uma repetição máxima (1RM) e 20RM. O protocolo de treinamento consistiu em: 3 sessões de exercícios (leg press, supino reto, mesa flexora, puxador frente), com intervalos de 1 minuto entre os exercícios e 2 minutos entre cada volta, totalizando 3 voltas por sessão. Cada sessão teve diferentes volumes (12, 16 e 20 repetições) e intervalo de 96 horas (h) entre as sessões. A dosagem da CK, a partir de uma amostra sanguínea, foi realizada em repouso, 24, 48 e 72h pós sessão. Os dados foram analisados pelo software STATISTIC 7, adotando significância de p<0,05. Resultados: Os grupos apresentaram pico de CK após $48 \mathrm{~h}$ ao repouso, não havendo diferença entre grupos, porém, verificou-se maiores concentrações de CK/repetições nas sessões próximas da falha concêntrica, que nos DT ocorreu na sessão de 16 repetições $(1,46 \pm 0,50)$, já nos TR ocorreu na sessão de 20 repetições $(1,71 \pm 0,62)$. Conclusão: As concentrações de CK não sofreram influência do nível de treinamento dos indivíduos, mas sim da proximidade a falha concêntrica, onde o pico de $48 \mathrm{~h}$ mostrou-se relevante para a prescrição do treinamento.

Palavras-chave: Creatina quinase; Exercícios em circuito; Força muscular; Treinamento físico. 


\begin{abstract}
Objective: To analyze the kinetics of creatine kinase $(\mathrm{CK})$ and its concentrations in resistance training in circuit, with different volumes of a fixed weight of twenty maximum repetitions (20RM), for trained (TR) and untrained (DT) men. Method: The sample comprised, by volunteering, 18 men (9 volunteers TR and 9 DT), aged between 18 and 35 years. After receiving information about the project, the volunteers, carry out a physical evaluation, and later tests and retests of a maximum repetition (1RM) and 20RM. The training protocol consisted of: 3 exercise sessions (leg press, bench press, flexing table, front puller), with intervals of 1 minute between exercises and 2 minutes between each lap, totaling 3 laps per session. Each session had different volumes (12, 16 and 20 repetitions) and a 96-hour (h) interval between sessions. The measurement of CK, from a blood sample, was done at rest, 24, 48 and $72 \mathrm{~h}$ after the session. The data were hanged by the STATISTIC 7 software, adopting a significance of $\mathrm{p}<0.05$. Results: The groups dissipated from CK after $48 \mathrm{~h}$ at rest, with no difference between groups, however, there were greater adjustments of CK/repetitions in the previous combinations of the concentric failure, which in DT occurred in the session of 16 repetitions (1.46 \pm 0.50$)$, no longer TR occurred in the 20 repetition session $(1.71 \pm 0.62)$. Conclusion: The CK concentrations were not influenced by the training level of the owners, but by the proximity to a concentric failure, where the peak of $48 \mathrm{~h}$ was relevant to the training prescription.
\end{abstract}

Keywords: Creatine kinase; Circuit exercises; Muscle strength; Physical training.

\title{
Resumen
}

Objetivo: Analizar la cinética de la creatina quinasa (CK) y sus recomendaciones en el entrenamiento de fuerza en circuito, con diferentes volúmenes de un peso fijo de veinte repeticiones máximas (20RM), para hombres entrenados (TR) y desentrenados (DT). Método: La muestra estuvo compuesta, de forma voluntaria, por 18 hombres (9 voluntarios TR y 9 DT), con edades comprendidas entre 18 y 35 años. Luego de recibir información sobre el proyecto, los voluntarios, realizan una evaluación física, y posteriormente pruebas y repruebas de una repetición máxima (1RM) y 20RM. El protocolo de entrenamiento consistió en: 3 sesiones de ejercicios (prensa de piernas, press de banca, mesa de flexión, tirador frontal), con intervalos de 1 minuto entre ejercicios y 2 minutos entre cada vuelta, totalizando 3 vueltas por sesión. Cada sesión tuvo diferentes volúmenes (12, 16 y 20 repeticiones) y un intervalo de 96 horas (h) entre sesiones. La medición de CK, a partir de una muestra de sangre, se realizó en reposo, 24, 48 y 72h después de la sesión. Los datos fueron colgados por el software STATISTIC 7, adoptando una significancia de p<0,05. Resultados: Los grupos se disiparon de $\mathrm{CK}$ a las $48 \mathrm{~h}$ de reposo, sin diferencia entre grupos, sin embargo, hubo mayores ajustes de $\mathrm{CK} /$ repeticiones en las combinaciones previas del fallo concéntrico, lo que en DT ocurrió en la sesión de 16 repeticiones $(1,46 \pm 0,50)$, ya no se presentó TR en la sesión de 20 repeticiones $(1,71 \pm 0,62)$. Conclusión: Las concentraciones de CK no fueron influenciadas por el nivel de entrenamiento de los individuos, sino por la proximidad a una falla concéntrica, donde el pico de $48 \mathrm{~h}$ fue relevante para la prescripción de entrenamiento.

Palabras clave: Creatina quinasa; Ejercicios de circuito; Fuerza muscular; Entrenamiento físico.

\section{Introdução}

O treinamento resistido caracterizado pela ação muscular realizada com a finalidade de executar movimentos contra uma determinada resistência (Fleck \& Kraemer, 2017), por meio de suas variáveis - volume, intensidade e velocidade de execução; pode provocar modificações de ordem hormonal e estrutural no músculo esquelético (Clarkson \& Hubal, 2002; Kraemer \& Ratamess, 2005; Prestes et al., 2016). Essas modificações ou danos no tecido muscular, em resposta a fatores bioquímicos e sobrecarga mecânica decorrentes do exercício físico, normalmente são provocadas por desordens nas estruturas das fibras musculares - membranas, linha Z, sarcolema, túbulos T e miofibrilas; resultando no aumento da permeabilidade da membrana, permitindo o extravasamento de substâncias para o meio extracelular (Friden \& Lieber, 1992; Clarkson \& Newham, 1995; Foschini, Prestes \& Charro, 2007; Schneider, Périco \& Pozzobon, 2015; Barros et al., 2020).

Neste contexto, existem algumas enzimas ou proteínas que se caracterizam como marcadores de danos musculares, como: Creatina quinase (CK), Lactato desidrogenase (LDH), fragmentos da cadeia pesada de miosina (MHC), troponina-I e mioglobina; que mediante aos danos nas estruturas musculares extravasam para a corrente sanguínea aumentando suas concentrações séricas (Brown, Child \& Donnelly, 1997; Willoughby, McFarlin \& Bois, 2003; Neto, Almeida \& Campos, 2017). Dentre todas estas moléculas citadas anteriormente, a CK é descrita como o melhor marcador indireto de danos teciduais no músculo esquelético após o exercício, especialmente aqueles com predominância excêntrica (Smith et al., 1994; Nosaka et al., 2005; Figueira et al., 2017; Barros et al., 2020). 
Assim, a CK é uma proteína dimérica composta por uma subunidade B e M que são subdivididas em CK-BB - maior prevalência no cérebro (Lang \& Wurzburg, 1982; Williams, Kreider \& Branch, 2000); CK-MB - maior prevalência no miocárdio (Frederiks et al., 2002); e CK-MM - maior prevalência no músculo esquelético (Apple, Hellsten \& Clarkson, 1988). Normalmente, as concentrações de CK no soro aumentam em poucas horas (h) após o treinamento resistido (Neto et al., 2007; Fortunato et al., 2018). A partir de intervalos de repouso normais de 60-400 unidade por litro (U/L), a CK aumenta em 100\% dentro de 8 h após o exercício resistido e continua aumentando atingindo valores que variam de 300-6000 U/L, sendo os níveis máximos identificados entre a 24 e 96h após o exercício inicial (Schlattner, Tokarska-Schlattner \& Wallimann, 2006; Brancario et al., 2008). Essas alterações na concentração de CK são altamente variáveis, podendo ser afetadas por fatores individuais e variáveis do treinamento (Machado \& Willardson, 2010; Carmo, Pereira \& Machado, 2011; Podgórski et al., 2020).

Desse modo, o treinamento máximo, ou que leva até a falha concêntrica (incapacidade de realizar a próxima repetição), tem sido relacionado ao aumento da ativação de unidades motoras (Rooney, Herbert \& Balnave, 1994; Drinkwater et al., 2005) e alto estresse mecânico, com expressão gênica da CK associada aos danos e reparos do processo muscular (Goldspink et al., 1992), onde aumentos na concentração de CK podem ser utilizados como indicativos de lesão muscular esquelética em indivíduos sem enfermidades (Foschini, Prestes \& Charro, 2007). Ademais, tem-se sugerido na literatura, que a fadiga após o treinamento até a falha pode aumentar os riscos de overtraining e lesões (Willardson, 2007; Willardson, Norton \& Wilson, 2010).

Portanto, a fim de averiguar qualquer aumento no estresse muscular ou a tolerância do indivíduo ao exercício muscular, a determinação da concentração sanguínea de CK parece ser um parâmetro sensível e confiável (Hartmann \& Mester, 2000). No entanto, não foram encontrados estudos analisando o dano muscular pelas concentrações de CK em um mesmo protocolo e peso fixo, em diferentes volumes. Sendo assim, o presente estudo tem como objetivo analisar a cinética da CK e suas concentrações no treinamento resistido em circuito, com diferentes volumes de um peso fixo de vinte repetições máximas (20RM), para homens treinados (TR) e destreinados (DT).

\section{Metodologia}

\subsection{Caracterização da pesquisa e da amostra}

Este estudo se caracteriza como uma pesquisa de abordagem quantitativa, do tipo transversal (Pereira et al., 2018). De acordo com Cohen (1992), para um nível $\alpha$ de 0,05 e poder da amostra de 0,91 (91\%), o tamanho amostral exigido foi de oito indivíduos por grupo para um tamanho do efeito de 0,60. Sendo assim, participaram do estudo 18 voluntários adultos do sexo masculino com idade entre 18 e 35 anos, subsequentemente divididos em grupo TR: constituído por 9 indivíduos com mais de um ano de treinamento; e em grupo DT: formado por 9 indivíduos que não possuíam experiência em treinamento; tendo como características gerais as respectivas informações demonstradas na Tabela 1.

Tabela 1. Características gerais dos voluntários (TR e DT) do presente estudo.

\begin{tabular}{lcc}
\hline \multirow{2}{*}{ Parâmetros } & Grupo TR $(\mathbf{n}=\mathbf{9})$ & Grupo DT $(\mathbf{n}=9)$ \\
\cline { 2 - 3 } & Média \pm Desvio Padrão & Média \pm Desvio Padrão \\
\hline Idade (anos) & $27,55 \pm 4,37$ & $28,00 \pm 5,78$ \\
Estatura (cm) & $173,88 \pm 5,15$ & $173,50 \pm 9,78$ \\
Peso corporal $(\mathbf{K g})$ & $77,77 \pm 8,78$ & $81,60 \pm 20,89$ \\
IMC $(\%)$ & $25,76 \pm 2,85$ & $26,36 \pm 5,75$ \\
\hline
\end{tabular}

Legenda: TR: treinado; DT: destreinado; n: números; cm: centímetros; Kg: quilogramas; IMC: Índice de Massa Corpora; \%: percentual.

Fonte: Autores. 
Foram adotados como critérios de inclusão: (a) os indivíduos deveriam ser do sexo masculino; (b) ter idade entre 18 e 35 anos; (c) praticar de forma regular treinamento resistido por período superior a um ano - grupo TR; e (d) não possuir experiência em treinamento resistido - grupo DT. Em contra partida, os critérios de exclusão adotados foram: (a) uso de esteroides anabólicos androgênicos nos seis meses que precederam o estudo; (b) presença de alguma doença metabólica que pudesse alterar os resultados do estudo; (c) alguma patologia ou lesão que limitasse a execução total ou parcial do protocolo de treinamento; (d) presença de problemas cardíacos, respiratórios, musculares, como alguma lesão ou cirurgia recente; e (e) indivíduos fumantes. Os voluntários foram orientados a manterem o mesmo padrão dietético, evitando alimentos e bebidas estimulantes nas 24 horas que precederam os testes, tão pouco realizar exercícios físicos nos três dias que antecederam cada intervenção, esta que teve os mesmos horários fixados.

\subsection{1 Ética}

Em consonância com as normas que regulamentam a pesquisa com seres humanos (Resolução 466/12 do Conselho Nacional de Saúde), este projeto foi submetido e aprovado pelo Comitê de Ética e Pesquisa em Seres Humanos da Universidade Federal de São Carlos - UFSCar (CAAE: 59303416.1.0000.5504). Todos os procedimentos foram realizados no Laboratório de Fisiologia do Exercício do Departamento de Ciências Fisiológicas da UFSCar.

\subsection{Desenho experimental}

Esta pesquisa avaliou as concentrações da $\mathrm{CK}$ em um protocolo de treinamento resistido em circuito com diferentes volumes (12, 16 e 20 repetições) nos exercícios e respectiva ordem: leg press (LP), supino reto (SR), mesa flexora (MF) e puxador frente (PF); com um peso fixo equivalente a 20RM, que foi determinado a partir do teste e reteste de uma repetição máxima (1RM), e posteriormente, teste e reteste de 20RM. As coletas de dados foram realizadas pré sessão, 24, 48 e 72h após cada sessão. Desse modo, o intervalo entre uma sessão e outra foi de $96 \mathrm{~h}$.

\subsection{Procedimentos experimentais}

\subsubsection{Avaliação dos voluntários}

Inicialmente, os voluntários receberam informações sobre todo o desenvolvimento do projeto, como: objetivos, riscos, benefícios, delineamento do protocolo e intervenção; sendo entregue àqueles que estivessem de acordo com a realização e participação nesta pesquisa, o Termo de Consentimento Livre e Esclarecido (TCLE), o qual deveria ser assinado pelo voluntário. Posteriormente, os voluntários foram avaliados fisicamente - peso corporal e estatura; através de uma balança antropométrica mecânica modelo 104 A (Welmy ${ }^{\circledR}$, Santa Barbara D’Oeste, Brasil), com precisão de 100 gramas e capacidade de 300 quilogramas $(\mathrm{Kg})$, e escala de precisão de 0,1 centímetros (cm). O Índice de Massa Corporal (IMC) foi determinado pela razão do peso pela estatura ao quadrado $\left(\mathrm{IMC}=\right.$ peso/estatura $\left.{ }^{2}\right)$.

\subsubsection{Testes máximos}

Na segunda etapa da pesquisa todos os voluntários realizaram duas sessões de familiarização, afim de refinar o padrão de movimento pré-estabelecido, e posteriormente testes de 1RM e 20RM nos exercícios LP, SR, MF e PF. O teste de 1RM foi embasado no protocolo de Brown e Weir (2001) que consistiu em: aquecimento com 8 repetições a 50\% de 1RM estimada, e intervalo de 2 minutos, seguido de uma série com 70\% de 1RM. A partir disso iniciou a primeira tentativa para determinação de 1RM com o mínimo de 3 e máximo de 5 tentativas, com intervalos de 5 minutos entre as tentativas. Foi realizado o reteste no período de 48h, caso houvesse diferença igual ou superior a 5\% um terceiro teste seria realizado. Para o teste de 20RM, de acordo com Shimano et al. (2006) foi estimado o peso de aproximadamente 55 a 60\% de 1RM para que os indivíduos executassem 19 
repetições sendo a $20^{\mathrm{a}}$ repetição a máxima. Quando as repetições eram subestimadas ou superestimadas, o peso era reajustado para que houvesse outra tentativa com o máximo de 2; e intervalos de 5 minutos. Assim como no teste de 1RM, também foi realizado o reteste após $72 \mathrm{~h}$, caso houvesse diferença igual ou superior a $5 \%$ um terceiro teste seria realizado.

\subsubsection{Protocolo de treinamento}

O método de treinamento em circuito alternado por segmento nos exercícios LP, SR, MF e PF com intervalos de 1 minuto entre os exercícios e 2 minutos entre cada volta, em um total de 3 voltas por sessão, foi adotado como protocolo de intervenção nesta pesquisa. Os voluntários realizaram as 3 sessões de treinamento com diferentes volumes (12, 16 e 20 repetições) do peso encontrado para execução de 20RM e intervalo de 96h entre as sessões.

\subsubsection{Concentrações da creatina quinase}

As análises das concentrações de CK foram realizadas através da coleta de sangue arterializado da polpa digital, devidamente assepsiada com álcool $70 \%$ e limpa para retirada de possíveis resíduos. A primeira gota foi descartada para evitar qualquer tipo de influência no teste. Em seguida, foi coletada uma amostra de 30 $\mu \mathrm{L}$ (microlitros) de sangue com auxílio de uma pipeta, seguida da aplicação dessa amostra em uma fita reagente de CK do equipamento Reflotron (Roche®, Berlim, Alemanha). O valor é apresentado em U/L. Para a determinação da cinética da CK, o sangue foi coletado pré e pós exercício em $24 \mathrm{~h}$, $48 \mathrm{~h}$ e 72h de cada sessão de treino. Já a CK/repetição foi determinada a partir do delta CK do pico em relação ao repouso (pico repouso) dividido pelas repetições realizadas.

\subsection{Análise estatística}

Os dados foram tratados por procedimentos estatístico compatíveis com os objetivos propostos utilizando o software STATISTIC 7 (Statsoft inc. Tulsa. USA). Inicialmente foi realizada a verificação da normalidade dos dados pelo teste de Kolmogorov- Smirnov e homogeneidade por Levene. Os testes posteriores foram realizados de acordo com a normalidade dos dados, teste de variância Anova Two-Way, e para identificar as diferenças das sessões de cada grupo foi utilizado o teste de Posthoc Tukey, para as variáveis paramétricas e Kruskal Wallis Anova, para as variáveis não paramétricas. Para as conclusões das análises estatísticas foi utilizado o nível de significância de $5 \%(\mathrm{p}<0,05)$.

\section{Resultados}

O número de repetições realizadas para os grupos foi: sessão de 12 repetições (DT = 144,00 \pm 0,00; e TR =144,00 \pm 0,00); sessão de 16 repetições (DT $=172,00 \pm 18,80$; e $\mathrm{TR}=187,67 \pm 5,50$; e sessão de 20 repetições $(\mathrm{DT}=182,33 \pm 23,10$; e $\mathrm{TR}=192,00 \pm 20,18$ ), havendo diferença estatística significativa, $\mathrm{p}<0,05$, entre TR 12 e TR 16 repetições, TR 12 e TR 20 repetições, DT 12 e DT 20 repetições. Ademais, a cinética da CK (U/L) apresentou diferença somente no pico de 48h em relação ao repouso, sendo tal característica observada em ambos os grupos, DT e TR, em todas as sessões: DT 12 repetições (Repouso $=105,24 \pm 31,62 \mathrm{U} / \mathrm{L} ; 48 \mathrm{~h}=189,50 \pm 55,69 \mathrm{U} / \mathrm{L})$, DT 16 repetições (Repouso = 102,94 $\pm 30,36 \mathrm{U} / \mathrm{L} ; 48 \mathrm{~h}=351,82 \pm 83,84$ $\mathrm{U} / \mathrm{L}$ ), DT 20 repetições (Repouso $=106,03 \pm 34,25 \mathrm{U} / \mathrm{L} ; 48 \mathrm{~h}=312,10 \pm 85,10 \mathrm{U} / \mathrm{L})$ e TR 12 repetições $($ Repouso $=113,25 \pm$ 27,90 U/L; 48h = 268,66 \pm 28,84 U/L), TR 16 repetições (Repouso = 108,78 \pm 22,77 U/L; 48h = 330,88 \pm 89,01 U/L), TR 20 repetições (Repouso $=102,96 \pm 19,82 \mathrm{U} / \mathrm{L} ; 429,55 \pm 101,34 \mathrm{U} / \mathrm{L})$; conforme demonstra a Figura 1, cujo a qual apresenta a cinética das concentrações de CK em repouso, 24h, 48h e 72h do grupos TR e DT nas sessões 12, 16 e 20 repetições. 
Figura 1. Cinética das concentrações de CK em Repouso, 24h, 48h e 72h A) DT nas sessões 12, 16 e 20 repetições. B) TR nas sessões 12, 16 e 20 rep. Para esta análise foi utilizado o teste não paramétrico de Kruskal Wallis Anova. Os dados estão apresentados em média \pm desvio padrão. ${ }^{*} \mathrm{p}<0,05$, diferença entre DT e TR em 12,16 e 20 rep. \#p<0,05, diferença entre DT 12 e DT 16 rep., diferença entre TR 12 e TR 16 rep.; +p<0,05, diferença entre DT 12 e DT 20 rep., diferença entre TR 12 e TR 20 rep. Legenda: CK: creatina quinase; U/L: unidade por litro; TR: treinados; DT: destreinados; rep.: repetições; h: horas.

A)

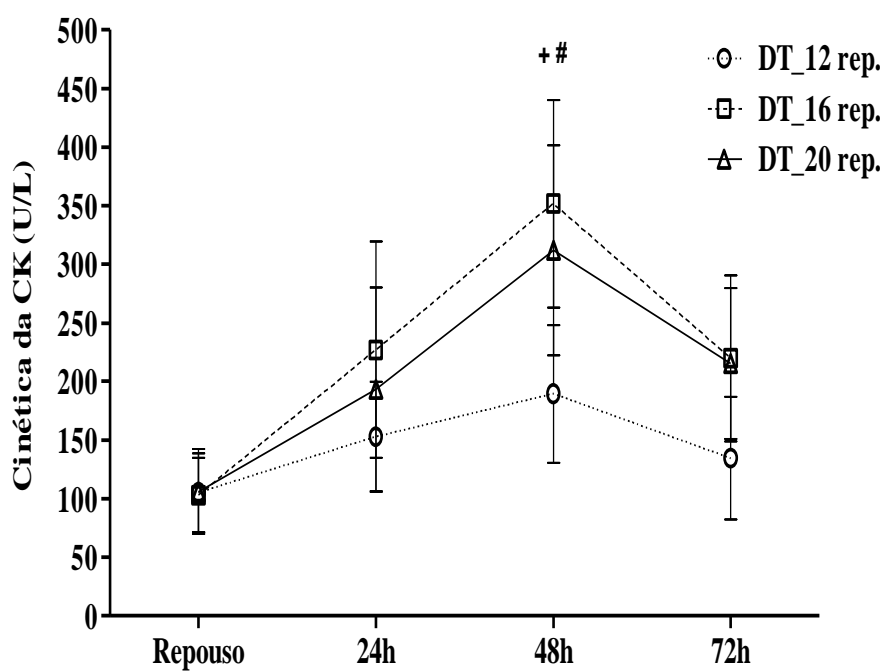

B)

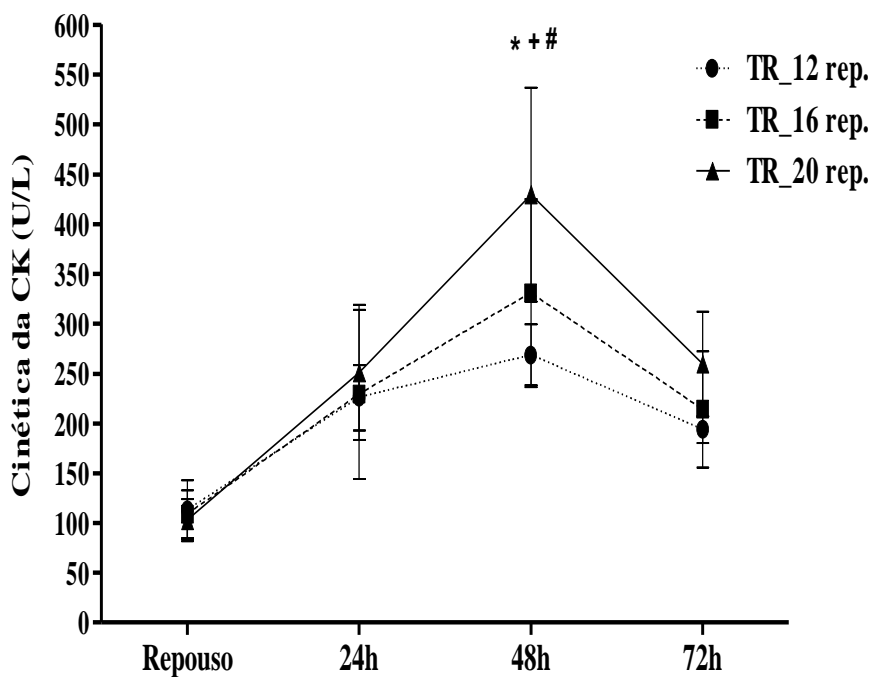

Fonte: Autores.

Em continuação, quando analisamos o pico de 48h da CK (U/L) para os grupos avaliados (Figura 2) nas três sessões, os resultados obtidos para cada sessão para DT e TR, respectivamente foram: 12 repetições $(\mathrm{DT}=189,5 \pm 55,69$ U/L; e TR = 268,66 \pm 28,84 U/L), 16 repetições $(\mathrm{DT}=51,82 \pm$ 83,84 U/L; e TR = 330,88 \pm 89,01 U/L), e 20 repetições $(\mathrm{DT}=312,1 \pm 85,10$ U/L; e 429,55 — 101,34 U/L); através dos quais foi possível verificar a existência de diferença estatística significativa, p<0,05, entre DT 12 e DT 16 repetições, DT 12 e DT 20 repetições, TR 12 e TR 20 repetições, e entre os grupos na sessão de 20 repetições.

Figura 2. Pico da CK em 48h nas sessões de 12, 16 e 20 rep. para indivíduos treinados e destreinados. Para esta análise foi utilizado o teste paramétrico Post-hoc Tukey. Os dados estão apresentados em média \pm desvio padrão. *p<0,05, diferença entre DT e TR em 12, 16 e 20 repetições. " p<0,05, diferença entre DT 12 e DT 16 repetições, diferença entre TR 12 e TR 16 repetições; ${ }^{+} \mathrm{p}<0,05$, diferença entre DT 12 e DT 20 repetições, diferença entre TR 12 e TR 20 repetições. Legenda: CK: creatina quinase; U/L: unidade por litro; rep.: repetições; H: horas.

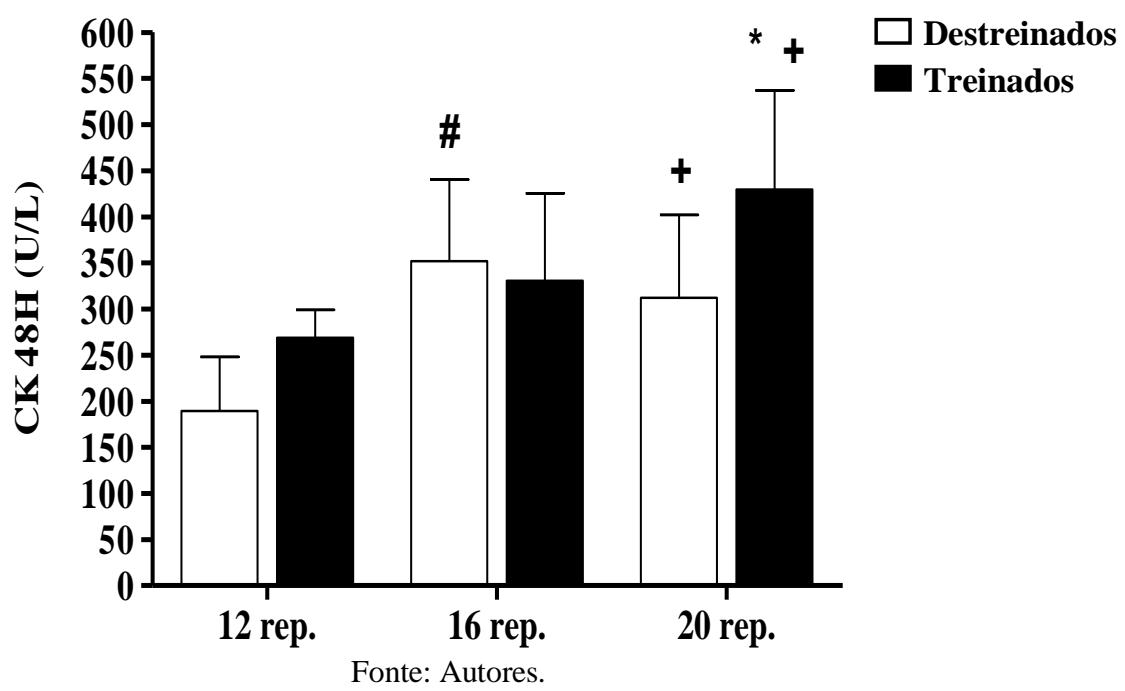


Por fim, ao analisarmos a concentração de CK em cada repetição verificamos os seguintes valores em cada sessão para os grupo DT e TR, respectivamente: 12 repetições $(\mathrm{DT}=0,59 \pm 0,39$; e TR = 1,08 $\pm 0,32), 16$ repetições $(\mathrm{DT}=1,46 \pm 0,50$; e $\mathrm{TR}=1,18 \pm 0,48)$ e 20 repetições ( $\mathrm{DT}=1,12 \pm 0,32$; e $\mathrm{TR}=1,71 \pm 0,62$ ), através dos quais foi possível verificar a existência de diferença estatística significativa, p<0,05, entre DT 12 e DT 16 repetições; DT 12 e DT 20 repetições; TR 12 e TR 20 repetições e entre os grupos na sessão de 20 repetições.

Figura 3. $\Delta \mathrm{CK}$ em $48 \mathrm{~h}$ por repetição nas sessões de 12, 16 e 20 rep. nos grupos Destreinados e Treinados. Para esta análise paramétrica, foi utilizado o teste de Post-hoc Tukey. Os dados estão apresentados em média \pm desvio padrão. ${ }^{*}$ p $<0,05$, diferença entre DT e TR em 12, 16 e 20 repetições. " $\mathrm{p}<0,05$, diferença entre DT 12 e DT 16 repetições, diferença entre TR 12 e TR 16 repetições; ${ }^{+} \mathrm{p}<0,05$, diferença entre DT 12 e DT 20 repetições, diferença entre TR 12 e TR 20 repetições. Legenda: $\Delta C K$ : concentração de creatina quinase; U/L: unidade por litro; rep.: repetições; H: horas.

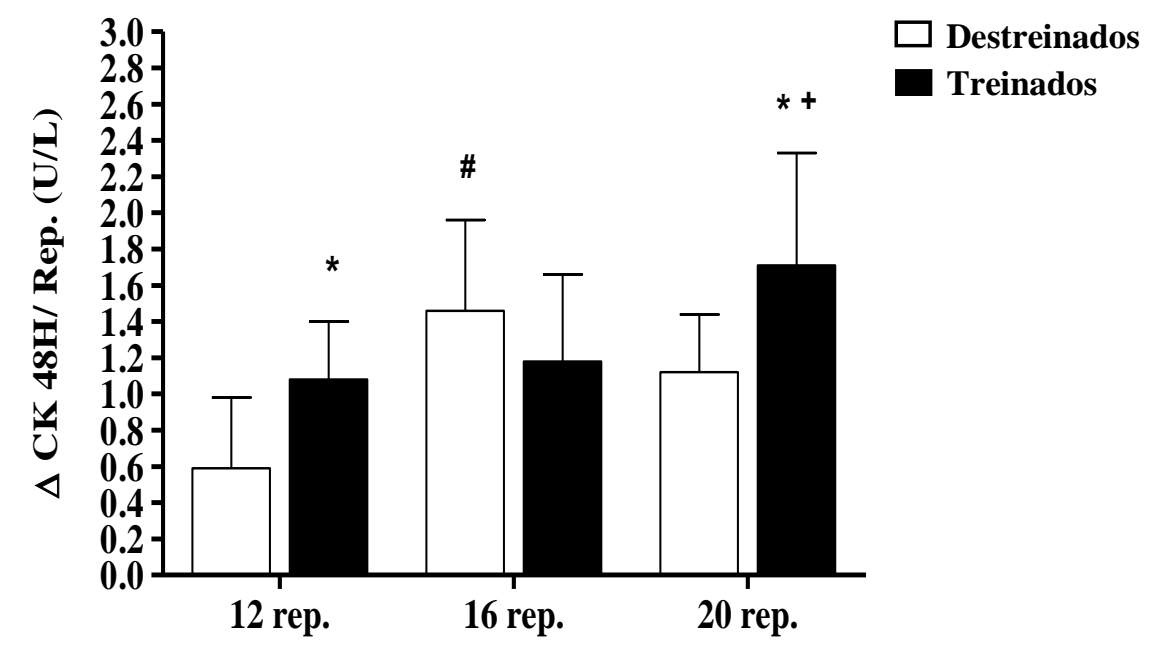

Fonte: Autores.

\section{Discussão}

Diante do objetivo de analisar a cinética da CK e suas concentrações no treinamento resistido em circuito, com diferentes volumes de um peso fixo de 20RM, para homens TR e DT, este estudo teve como principal descoberta, que o estado físico prévio dos indivíduos, TR e DT, não acarretou em diferenças significativas nos níveis séricos de CK entre os grupos avaliados. Resultado inesperado, visto que alguns estudos têm relatado o efeito protetor - atenuação nos sintomas e ocorrência dos danos musculares quando realizada uma segunda sessão de mesmo volume e intensidade (Lima \& Denadai, 2011); de um treinamento prévio (Clarkson \& Tremblay, 1988; Nosaka \& Clarkson, 1995; Nosaka \& Newton, 2002; Newton et al., 2008).

Neste contexto, Nosaka \& Newton (2002) relataram em seu estudo que o dano muscular não foi exacerbado após o exercício excêntrico máximo, quando precedido por 8 semanas de treinamento concêntrico. Por sua vez, Newton et al. (2008) verificaram efeito protetor em indivíduos que treinaram previamente antes de realizarem ações musculares concêntricas e excêntricas. Este resultado é semelhante ao mostrado em outros estudos que relatam o "efeito repetido" usando sujeitos não treinados (Clarkson \& Tremblay, 1988; Nosaka \& Clarkson, 1995). Os resultados desta pesquisa podem ter sido diferentes aos encontrados nos estudos anteriores, em virtude dos indivíduos, apesar de treinados, não estarem adaptados ao treinamento de resistência muscular determinado no protocolo experimental do presente estudo.

Assim, ao analisarmos a cinética das concentrações da $\mathrm{CK}$, verificamos a ocorrência de um pico no período referente à 48h após as sessões para ambos os grupos, o que também foi observado por Smith et al. (1994) em um protocolo de 3 séries de 12 repetições à $80 \%$ de $1 \mathrm{RM}$ e por Lima e Denadai (2011) em um protocolo de 4 séries de 12 repetições máximas, divergindo dos estudos realizados com indivíduos jovens do sexo masculino executados por Santos et al. (2019), Forte, Paiva e Meireles 
(2020) e Barros et al. (2020) os quais verificaram que o pico da concentração de CK ocorreu após 24h da realização da respectiva sessão de treinamento resistido.

No entanto, em outro estudo que objetivou avaliar protocolos com diferentes intervalos de repouso ( 1 e 3 minutos), com 3 séries à $80 \%$ de 1RM, com 5 exercícios de membros superiores, Rodrigues et al. (2010) observou-se um pico no momento relativo à 48h nas concentrações de CK para os dois protocolos propostos em sua pesquisa. Machado e Willardson (2010), por sua vez, ao analisar indivíduos que responderam de forma diferente ao dano muscular quanto aos níveis de CK, verificaram que para 3 séries de 10 repetições com intensidade de 10RM, em 6 exercícios, com 1 minuto de intervalo entre as séries, os indivíduos que apresentaram maior resposta atingiram picos em 48 e 72h. Ou seja, o período de 48h após uma sessão de treinamento, em protocolos semelhantes aos do presente estudo, parece ser relevante e deve ser levado em consideração na prescrição e periodização do treinamento.

Em continuação, de acordo com Rodrigues et al. (2010), e subsequentemente, Neto, Almeida \& Campos (2017), protocolos de treinamento resistido que envolvem intensidades moderadas à altas, com performances que cheguem até a falha concêntrica, induzem significantemente o aumento da concentração de CK e maiores danos às fibras musculares, sendo aquela utilizada como indicador de uma possível futura lesão, e também para monitorar a carga de treinamento. Tal característica fisiológica, causada pelo protocolo de treinamento resistido, pode ter ocorrido neste estudo ao verificarmos que a proximidade da falha concêntrica das sessões afetou as concentrações de CK. Este cenário no grupo DT foi evidenciado entre as sessões de 16 e 20 repetições, onde as concentrações de CK foram maiores, e a sessão de 12 repetições. Por outro lado, no grupo TR esta superioridade foi evidenciada na sessão de 20 repetições em relação a sessão de 12 repetições.

Essa diferença entre os grupos pode ter ocorrido devido a sessão de 16 repetições ter exigido maior recrutamento muscular para o grupo DT, enquanto que para o grupo TR isso só tenha ocorrido na sessão de 20 repetições. Diante desses resultados, e com base em estudos anteriores, sabemos que indivíduos TR de forma crônica apresentam ganhos de força decorrentes da junção de adaptações neurológicas, endocrinológicas, intramusculares e intermusculares (Fry, 2004; Kraemer \& Ratamess, 2005; Podgórski et al., 2020). Isto indica uma maior capacidade do grupo TR em suportar cargas de treinamento mais intensas, devido à todas as adaptações provocadas pelo treinamento, em relação ao grupo DT (Teixeira et al., 2019), e fortalece o discurso sobre a importância da realização de exercícios físicos resistidos para a promoção da saúde e desempenho esportivo (Barroso, Tricoli \& Ugrinowitsch, 2005; Basilio et al., 2017; Rödel et al., 2020).

Por conseguinte, este fato foi confirmado ao corrigirmos uma limitação do presente estudo com relação a diferença de volume entre as sessões, através da determinação das concentrações de CK por repetições realizadas, em que diferentemente da análise da CK de 48h onde as sessões de 16 e 20 repetições foram superiores a sessão de 12 repetições, quando corrigido pelas repetições realizadas somente a sessão de 16 repetições foi maior que a sessão de 12 repetições para o grupo DT. Já o grupo TR, mesmo quando corrigimos pelas repetições realizadas, a concentração de CK foi maior na sessão de 20 repetições em relação a sessão de 12 repetições, assim como na análise anterior do pico de $48 \mathrm{~h}$. Assim, ressaltamos que esta pesquisa seguiu rigorosamente seu objetivo, de modo a ofertar resultados relevantes a temática abordada, contribuindo para a realização de futuros estudos e intervenções.

\section{Conclusão}

O presente estudo demonstrou que neste protocolo de treinamento resistido em circuito, o nível de treinamento não afetou as concentrações de CK, além de ambos os grupos, nas diferentes sessões apresentarem picos em 48h, período este que parece ser relevante e deve ser levado em consideração na prescrição e periodização do treinamento. Outro fator observado foi que a proximidade da falha concêntrica resultou em maiores concentrações de CK/repetições, sendo que para o grupo DT a falha 
ocorreu na sessão de 16 repetições, enquanto para os TR na sessão de 20 repetições. Adicionalmente, incentivamos a realização de novos estudos sobre a temática abordada e sugerimos a ampliação amostral, incluindo indivíduos do sexo feminino com intuito de observar a cinética da CK em outras populações, detectando características e produzindo conhecimentos que possibilitem a melhora da prescrição do treinamento para cada grupo, além da ampliação das faixas de intensidade, análises de outros marcadores e maior controle da densidade do treinamento.

\section{Agradecimentos}

Ao Prof. Dr. Sérgio Eduardo de Andrade Perez pelo auxílio na elaboração deste artigo, disponibilização de seu laboratório de pesquisa e alguns recursos materiais importantes à execução deste trabalho, e a todos os pesquisadores e voluntários que colaboraram com a realização deste estudo.

\section{Referências}

Apple, F. S., Hellsten, Y., \& Clarkson, P. M. (1988). Early detection of skeletal muscle injury by assay of creatine kinase MM isoforms in serum after acute exercise. Clinical chemistry, 34(6), 1102-1104. https://doi.org/10.1093/clinchem/34.6.1102

Barros, N. D. A., Aidar, F. J., Matos, D. G., Souza, R. F., Neves, E. B., Cabral, B. G. D. A. T., \& Reis, V. M. (2020). Evaluation of Muscle Damage, Body Temperature, Peak Torque, and Fatigue Index in Three Different Methods of Strength Gain. International journal of exercise science, $13(3)$, 1352-1365. https://pubmed.ncbi.nlm.nih.gov/33042368/

Barroso, R., Tricoli, V., \& Ugrinowitsch, C. (2005). Adaptações neurais e morfológicas ao treinamento de força com ações excêntricas. Revista brasileira de ciência e movimento, 13(2), 111-122. https://pesquisa.bvsalud.org/portal/resource/pt/lil-524821

Basilio, P. G., Tajes, R. T., Torres Filho, A. D., \& de Medeiros Lima, L. E. (2017). Efeitos benéficos das ações excêntricas no treino resistido. Revista Brasileira de Fisiologia do Exercício, 16(1), 34-39. https://doi.org/10.33233/rbfe.v16i1.1121

Brancaccio, P., Maffulli, N., Buonauro, R., \& Limongelli, FM (2008). Monitoramento de enzimas séricas em medicina esportiva. Clinics in sports medicine, 27(1), 1-18. https://doi.org/10.1016/j.csm.2007.09.005

Brown, L. E., \& Weir, J. P. (2001). Asep Procedures Recommendation I - Accurate Assessment of Muscular Strength and Power. Journal of Exercise Physiology Online. 4(3), 1-21. https://www.researchgate.net/publication/235782389_ASEP_Procedures_recommendation_I_Accurate_assessment_of_muscular _strength_and_power

Brown, S. J., Child, R. B., Day, S. H., \& Donnelly, A. E. (1997). Exercise-induced skeletal muscle damage and adaptation following repeated bouts of eccentric muscle contractions. Journal of sports sciences, 15(2), 215-222. https://doi.org/10.1080/026404197367498

Carmo, F. C., Pereira, R., \& Machado, M. (2011). Variability in resistance exercise induced hyperCKemia. Isokinetics and Exercise Science, 19(3), 191-197. https://doi.org/10.3233/IES-2011-0416

Clarkson, P. M., \& Hubal, M. J. (2002). Exercise-induced muscle damage in humans. American journal of physical medicine \& rehabilitation, 81(11), S52-S69. https://journals.lww.com/ajpmr/Fulltext/2002/11001/Exercise_Induced_Muscle_Damage_in_Humans.7.aspx

Clarkson, P. M. \& Newham, D. J. (1995). Asssociations between muscle soreness, damage, and fatigue. In: Gandevia, S. C., Enoka, R. M., McComas, A. J., Stuart, D. G., Thomas, G. K., \& Pierce, P. A. (eds). Fatigue. Advances in experimental medicine and biology, vol 384. Springer, Boston, MA. https://doi.org/10.1007/978-1-4899-1016-5_35.

Clarkson, P. M., \& Tremblay, I. S. A. B. E. L. L. E. (1988). Exercise-induced muscle damage, repair, and adaptation in humans. Journal of Applied Physiology, 65(1), 1-6. https://doi.org/10.1152/jappl.1988.65.1.1

Cohen, J. (1992). A power primer. Psychological Bulletin, 112(1), 155-159. https://doi.org/10.1037/0033-2909.112.1.155

Drinkwater, E. J., Lawton, T. W., Lindsell, R. P., Pyne, D. B., Hunt, P. H., \& McKenna, M. J. (2005). Training leading to repetition failure enhances bench press strength gains in elite junior athletes. The Journal of Strength \& Conditioning Research, 19(2), 382-388. https://doi.org/10.1519 / R-15224.1

Figueira, T. G., Magosso, R. F., Campanholi-Neto, J., Carli, J. P., \& Robert-Pires, C. M. (2017). Creatine Kinase Response of Physically Active Young Men to One-and Two-Legged Cycling. Journal of Exercise Physiology Online, 20(3), 168-176. https://www.asep.org/asep/asep/JEPonlineJUNE2017_Figueira.pdf

Fleck, S. J., \& Kraemer, W. J. (2017). Fundamentos do treinamento de força muscular. (4a ed). Artmed.

Forte, L. D. M., Paiva, R. R., \& Meireles, C. L. S. (2020). Respostas agudas e tardias da creatina quinase após duas configurações distintas de treinamento resistido. Arquivos Brasileiros de Educação Física, 3(1), 15-21. https://doi.org/10.20873/abef.2595-0096.v2n2p1621.2020

Fortunato, A. K., Pontes, W. M., De Souza, D. M. S., Prazeres, J. S. F., Marcucci-Barbosa, L. S., Santos, J. M. M., ... \& Da Silva, A. N. (2018). Strength Training session induces important changes on physiological, immunological, and inflammatory biomarkers. Journal of immunology research, 2018 , 1-13. https://doi.org/10.1155/2018/9675216 
Foschini, D., Prestes, J., \& Charro, M. A. (2007). Relação entre exercício físico, dano muscular e dor muscular de início tardio. Revista Brasileira de Cineantropometria $e \quad$ Desempenho Humano, 9(1), 101-106. https://www.researchgate.net/profile/JonatoPrestes/publication/26455321_Relationship_between_physical_exercise_muscle_damage_and_delayedonset_muscle_soreness/links/56aff76508ae8e37214d14 75/Relationship-between-physical-exercise-muscle-damage-and-delayed-onset-muscle-soreness.pdf

Fredericks, S., Murray, J. F., Carter, N. D., Chesser, A. M., Papachristou, S., Yaqoob, M. M., \& Holt, D. W. (2002). Cardiac troponin T and creatine kinase MB content in skeletal muscle of the uremic rat. Clinical chemistry, 48(6), 859-868. https://doi.org/10.1093/clinchem/48.6.859

Friden J. A. N., \& Lieber R. L. (1992). Structural and mechanical basis of exercise-induced muscle injury. Medicine and Science in Sports and Exercise, 24(5), 521-530. https://www.researchgate.net/profile/Jan-Friden-2/publication/21585576_Structural_and_mechanical_basis_of_exercise-induced_muscle_injur y_Review/links/59 dbdb6daca2728e20183317/Structural-and-mechanical-basis-of-exercise-induced-muscle-injury-Review.pdf

Fry, A. C. (2004). The role of resistance exercise intensity on muscle fibre adaptations. Sports medicine, 34(10), 663-679. http://doi.org/10.2165/00007256200434100-00004.

Goldspink, G., Scutt, A., Loughna, P. T., Wells, D. J., Jaenicke, T., \& Gerlach, G. F. (1992). Gene expression in skeletal muscle in response to stretch and force generation. American Journal of physiology-regulatory, integrative and comparative physiology, 262(3), 356-363. https://doi.org/10.1152/ajpregu.1992.262.3.R356

Hartmann, U., \& Mester, J. (2000). Training and overtraining markers in selected sport events. Medicine and science in sports and exercise, 32(1), 209-215. https://doi.org/10.1097 / 00005768-200001000-00031

Kraemer, W. J., \& Ratamess, N. A. (2005). Hormonal responses and adaptations to resistance exercise and training. Sports medicine, 35(4), 339-361. http://doi.org/10.2165/00007256-200535040-00004.

Lang, H., \& Würzburg, U. (1982). Creatine kinase, an enzyme of many forms. Clinical chemistry, 28(7), 1439-1447. http://citeseerx.ist.psu.edu/viewdoc/download?doi=10.1.1.619.3097\&rep=rep1\&type=pdf

Lima, L. C. R. D., \& Denadai, B. S. (2011). The repeated bout effect: a comparison between upper and lower limbs. Motriz: Revista de Educação Física, 17(4), 738-747. https://doi.org/10.1590/S1980-65742011000400019

Machado, M., \& Willardson, J. M. (2010). Short recovery augments magnitude of muscle damage in high responders. Medicine and Science in Sports and Exercise, 42(7), 1370-1374. http://doi.org/10.1249/MSS.0b013e3181ca7e16

Neto, J. M. F. A., de Almeida, J. P. E., \& de Campos, M. F. (2017). Análise de marcadores celulares e bioquímicos sanguíneos para determinação de parâmetros de monitoramento do treinamento de praticantes de musculação. RBPFEX-Revista Brasileira de Prescrição e Fisiologia do Exercício, 11(70), 778-783. http://www.rbpfex.com.br/index.php/rbpfex/article/view/1266/982

Neto, J. M. F. A., Ferreira, D. C. B. G., dos Reis, I. C., Calvi, R. G., \& Rivera, R. J. B. (2007). Manutenção de microlesões celulares e respostas adaptativas a longo prazo no treinamento de força. Brazilian journal of biomotricity, 1(4), 1-16. https://www.redalyc.org/pdf/930/93010402.pdf

Newton, M. J., Morgan, G. T., Sacco, P., Chapman, D. W., \& Nosaka, K. (2008). Comparison of responses to strenuous eccentric exercise of the elbow flexors between resistance-trained and untrained men. The Journal of Strength \& Conditioning Research,22(2), 597-607. https://doi.org/10.1519/JSC.0b013e3181660003

Nosaka, K., \& Clarkson, P. M. (1995). Muscle damage following repeated bouts of high force eccentric exercise. Medicine and science in sports and exercise, 27(9), 1263-1269. https://europepmc.org/article/med/8531624

Nosaka, K., \& Newton, M. (2002). Repeated eccentric exercise bouts do not exacerbate muscle damage and repair. The Journal of Strength \& Conditioning Research, 16(1), 117-122. https://pubmed.ncbi.nlm.nih.gov/11834116/

Nosaka, K., Newton, M., Sacco, P., Chapman, D., \& Lavender, A. (2005). Partial protection against muscle damage by eccentric actions at short muscle lengths. Medicine and science in sports and exercise, 37(5), 746-753. http://doi.org/10.1249/01.MSS.0000162691.66162.00

Pereira, A. S., Shitsuka, D. M., Parreira, F. J., \& Shitsuka, R. (2018). Metodologia da pesquisa científica. UFSM. https://repositorio.ufsm.br/bitstream/handle/1/15824/Lic_Computacao_Metodologia-Pesquisa-Cientifica.pdf?sequence=1 .

Podgórski, T., Nowak, A., Domaszewska, K., Mączyński, J., Jabłońska, M., Janowski, J., \& Ogurkowska M. B. (2020). Muscle strength and inflammatory response to the training load in rowers. Peer-Reviewed \& Open Acess. 8(1), e10355. https://www.ncbi.nlm.nih.gov/pmc/articles/PMC7749653/

Prestes, J., Foschini, D., Marchetti, P., Charro, M., \& Tibana, R. (2016). Prescrição e periodização do treinamento de força em academias (2a ed). Manole.

Rodrigues, B. M., Dantas, E., de Salles, B. F., Miranda, H., Koch, A. J., Willardson, J. M., \& Simão, R. (2010). Creatine kinase and lactate dehydrogenase responses after upper-body resistance exercise with different rest intervals. The Journal of Strength \& Conditioning Research,24(6), 1657-1662. http://doi.org/10.1519/JSC.0b013e3181d8e6b1

Rooney, K. J., Herbert, R. D., \& Balnave, R. J. (1994). Fatigue contributes to the strength training stimulus. Medicine and science in sports and exercise, 26(9), 1160-1164. https://europepmc.org/article/med/7808251

Rödel, R. G. B., Danielevicz, A., de Moraes Sirydakis, M. E., \& Delevatti, R. S. (2020). Qualidade de vida associada à frequência semanal de treinamento: um estudo transversal comparativo. Research, Society and Development, 9(9), e663997549-e663997549. https://doi.org/10.33448/rsd-v9i9.7549

Santos, G. A., Moreira, S. R., Santos, F. R., \& Teixeira-Coelho, F. (2019). Efeito da ação muscular excêntrica na avaliação perceptual da dor muscular de início tardio. RBPFEX-Revista Brasileira De Prescrição E Fisiologia Do Exercício, 13(83), 472-480. http://www.rbpfex.com.br/index.php/rbpfex/article/view/1700 
Research, Society and Development, v. 10, n. 4, e56510414563, 2021

(CC BY 4.0) | ISSN 2525-3409 | DOI: http://dx.doi.org/10.33448/rsd-v10i4.14563

Schlattner, U., Tokarska-Schlattner, M., \& Wallimann, T. (2006). Mitochondrial creatine kinase in human health and disease. Biochimica et Biophysica Acta (BBA)-Molecular Basis of Disease, 1762(2), 164-180. https://doi.org/10.1016/j.bbadis.2005.09.004

Schneider, M., Périco, E., \& Pozzobon, A. (2015). Verificação do dano muscular através da avaliação da concentração de creatina quinase em indivíduos não atletas após prova de ciclismo não competitivo. Scientia Medica, 25(1), 19819-19819. https://doi.org/10.15448/1980-6108.2015.1.19819

Shimano, T., Kraemer, W. J., Spiering, B. A., Volek, J. S., Hatfield, D. L., Silvestre, R., \& Häkkinen, K. (2006). Relationship between the number of repetitions and selected percentages of one repetition maximum in free weight exercises in trained and untrained men. The Journal of Strength \& Conditioning Research, 20(4), 819-823. https://doi.org/10.1519/R-18195.1.

Smith, L. L., Fulmer, M. G., Holbert, D., McCammon, M. R., Houmard, J. A., Frazer, D. D., ... \& Israel, R. G. (1994). The impact of a repeated bout of eccentric exercise on muscular strength, muscle soreness and creatine kinase. British journal of sports medicine, 28(4), 267-271. http://doi.org/10.1136/bjsm.28.4.267

Teixeira, J. A. A., Magosso, R. F., Pires, C. M. R., Teixeira, K. K. L., Baldissera, V., \& de Andrade Perez, S. E. (2019). Determinação dos níveis de aptidão física para indivíduos treinados e destreinados a partir do Índice de Força Máxima Relativa (IFMR). RBPFEX-Revista Brasileira de Prescrição e Fisiologia do Exercício, 13(82), 182-188. http://www.rbpfex.com.br/index.php/rbpfex/article/view/1585

Willardson, J. M. (2007). The application of training to failure in periodized multiple-set resistance exercise programs. Journal of Strength and Conditioning Research, 21(2), 628-631. https://doi.org/10.1519/00124278-200705000-00058

Willardson, J. M., Norton, L., \& Wilson, G. (2010). Training to failure and beyond in mainstream resistance exercise programs. Strength \& Conditioning Journal, 32(3), 21-29. https://doi.org/10.1519/SSC.0b013e3181cc2a3a

Williams, M. H., Kreider, R. B., \& Branch, J. D. (2000). Creatina. Manole.

Willoughby, D. S., McFarlin, B., \& Bois, C. (2003). Interleukin-6 expression after repeated bouts of eccentric exercise. International journal of sports medicine, 24(1), 15-21. https://doi.org/10.1055/s-2003-37197 